\title{
GROWTH OF SOLUTIONS OF WEAKLY COUPLED PARABOLIC SYSTEMS AND LAPLACE'S EQUATION
}

\author{
N. A. WATSON
}

(Received 5 March 1985)

Communicated by R. O. Vyborny

\begin{abstract}
Let $u_{i}(x, t)$ be the $i$ th component of a nonnegative solution of a weakly coupled system of second-order, linear, parabolic partial differential equations, for $x \in \mathbf{R}^{n}$ and $0<t<T$. We obtain lower estimates, near $t=0$, for the Lebesgue measure of the set of $x$ for which $t^{\alpha / 2} u_{i}(x, t)$ exceeds 1 . Related results for Poisson integrals on a half-space are also described, some applications are given, and interesting comparisons emerge.
\end{abstract}

1980 Mathematics subject classification (Amer. Math. Soc.): 35 B 05; Secondary 31 B 05, 31 B 25, 35 B $30,35 \mathrm{~J} 05,35 \mathrm{~K} 45$.

\section{Introduction}

Let $\mu$ be a finite, positive Borel measure on $\mathbf{R}^{n}$. Let $w$ be the Poisson integral of $\mu$ on the half-space $\left.\mathbf{R}^{n} \times\right] 0, \infty[$, so that

$$
w(x, t)=\sigma_{n} \int_{\mathbf{R}^{n}} t\left(\|x-y\|^{2}+t^{2}\right)^{-(n+1) / 2} d \mu(y),
$$

where $\sigma_{n}$ is the reciprocal of

$$
\int_{\mathbf{R}^{n}}\left(\|y\|^{2}+1\right)^{-(n+1) / 2} d y
$$

Then $w$ is a harmonic function and, if $\mu$ is singular with respect to Lebesgue measure $m$ on $\mathbf{R}^{n}$, then $w(x, t) \rightarrow \infty$ as $t \rightarrow 0$ for $\mu$-almost every $x \in \mathbf{R}^{n}$. Recently, Ahern [1] obtained lower estimates for $m(\{x: w(x, t)>1\})$ near $t=0$.

(C) 1986 Australian Mathematical Society $0263-6115 / 86 \$ A 2.00+0.00$ 
In this paper we first extend his results to obtain estimates for $m\left(\left\{x: t^{\alpha} w(x, t)>\right.\right.$ 1\}), given a condition on $\mu$ which implies that $t^{\alpha} w(x, t) \rightarrow \infty$ as $t \rightarrow 0$ for $\mu$-almost all $x$ in some Borel set $A$; here $0 \leqslant \alpha<n$. We then present the corresponding estimates for solutions of certain weakly coupled systems of linear, second-order, parabolic partial differential equations. Of particular interest is the difference between two of the estimates. In the harmonic case, the lower estimate depends essentially on $\alpha$, while $\mu(A)$ appears as a multiplicative factor; in the parabolic case, $\alpha$ appears only as part of a multiplicative constant, and $\mu(A)$ is completely absent. Some applications of the estimates are also given, which differ significantly in form due to the difference in the estimates.

\section{The harmonic case}

For any $x, y \in \mathbf{R}^{n}$, we put $d(x, y)=\max \left\{\left|x_{1}-y_{1}\right|, \ldots,\left|x_{n}-y_{n}\right|\right\}$ and $\|x\|=$ $\left(x_{1}^{2}+\cdots+x_{n}^{2}\right)^{1 / 2}$, so that

$$
d(x, y) \leqslant\|x-y\| \leqslant n^{1 / 2} d(x, y) .
$$

The open cube $\left\{y \in \mathbf{R}^{n}: d(x, y)<r\right\}$, with centre $x$ and edge length $2 r$, is denoted by $Q(x, r)$.

Throughout this section, $\mu$ denotes a positive Borel measure on $\mathbf{R}^{n}$ such that

$$
\int_{\mathbf{R}^{n}}\left(1+\|x\|^{2}\right)^{-(n+1) / 2} d \mu(x)<\infty .
$$

This condition is necessary and sufficient for the Poisson integral $w$ of $\mu$ to be harmonic on $\left.\mathbf{R}^{n} \times\right] 0, \infty$, by Theorem 6 of [6].

Suppose that $0 \leqslant \alpha<n$, and that there is a Borel set $A$ such that

$$
\mu(\bar{Q}(x, r)) r^{\alpha-n} \rightarrow \infty \text { as } r \rightarrow 0
$$

$\mu$-a.e. on $A$. It then follows easily from the corollary to Theorem 2 of [2] that

$$
t^{\alpha} w(x, t) \rightarrow \infty \quad \text { as } t \rightarrow 0
$$

$\mu$-a.e. on $A$. It is therefore reasonable to seek a lower estimate for $m\left(\left\{x: t^{\alpha} w(x, t)\right.\right.$ $>1\})$ near $t=0$. Note that, if $\alpha=0$, then (1) holds $\mu$-a.e. on $\mathbf{R}^{n}$ if and only if $\mu$ is singular with respect to $m$. However, if $0<\alpha<n$ and (1) holds $\mu$-a.e. on $\mathbf{R}^{n}$, then $\mu$ is singular with respect to $(n-\alpha)$-dimensional Hausdorff measure (by Lemma 4 of [8]), but not conversely (see pages 19-21 of [8]). If $\alpha$ is a positive integer and $\mu$ is supported by a smooth surface of dimension $n-\alpha$, then $\mu(\bar{Q}(x, r)) r^{\beta-n} \rightarrow \infty$ as $r \rightarrow 0$, $\mu$-a.e. on $\mathbf{R}^{n}$, for every $\beta<\alpha$.

We use $C$, with various subscripts, to denote a positive constant which depends only on the subscripts; its value may vary from line to line. If $C$ is replaced by a Greek letter, the constant always has the same value. 
Our first result is an easy modification of Lemma 1 of [1].

Lemma 1. Suppose that $0 \leqslant \alpha<n$, and that (1) holds $\mu$-a.e. on a Borel set $A$ with $\mu(A)<\infty$. Then, if $\varepsilon>0$, there is a Borel set $E$ such that $\mu(A \backslash E)<\varepsilon$ and (1) holds uniformly on $E$.

In Lemma 1, we use a general Borel set $A$ instead of $\mathbf{R}^{n}$ partly because, for a given $\mu$, (1) may hold with different values of $\alpha$ on different sets. See also the proof of Theorem 3 below.

We recall Lemmas 2 and 3 of [1] for ease of reference.

Lemma 2. Suppose that $d(x, z) \leqslant r$, that $0<t \leqslant 2 r$, and that $w$ is the Poisson integral of $\mu$. Then there is a positive constant $\gamma_{n}$ such that

$$
w(x, t) \geqslant \gamma_{n} t r^{-n-1} \mu(\bar{Q}(z, r)) .
$$

LEMMA 3. If $\mathscr{F}$ is a finite collection of open cubes, then there is a subcollection $\mathscr{G}$ of disjoint cubes such that, for each $Q \in \mathscr{F}$, there is $Q(x, r) \in \mathscr{G}$ with $Q \subseteq$ $Q(x, 3 r)$.

Given any Borel set $A$, the modulus of continuity of $\mu$ over $A$ is defined by

$$
\omega_{A}(r)=\sup \{\mu(\bar{Q}(x, r)): x \in A\} .
$$

If $0 \leqslant \alpha<n$, and if $\gamma_{n}$ is the same as in (2), we put

$$
\delta_{\alpha, A}(t)=\inf \left\{r: \gamma_{n} t^{\alpha+1} r^{-n-1} \omega_{A}(r) \leqslant 1\right\}
$$

for all $t>0$.

With these notations, Theorem 1 of [1] can be extended in the following way.

THEOREM 1. Suppose that $0 \leqslant \alpha<n$, that (1) holds $\mu$-a.e. on a Borel set $A$ with $\mu(A)<\infty$, and that $w$ is the Poisson integral of $\mu$. Then there is $t_{0}>0$ such that

$$
m\left(\left\{x: t^{\alpha} w(x, t)>1\right\}\right) \geqslant C_{n} \mu(A) t^{\alpha+1} / \delta_{\alpha, A}(t)
$$

whenever $0<t \leqslant t_{0}$.

The proof of Theorem 1 differs from that of Ahern's result only in that $\mathbf{R}^{n}$ is replaced by $A$ and appropriate items in his argument are multiplied by $t^{\alpha}$; his auxiliary function $\delta(x, t)$ is replaced by $\delta_{\alpha}(x, t)=\inf \left\{r: \gamma_{n} t^{\alpha+1} r^{-n-1} \mu(\bar{Q}(x, r))\right.$ $\leqslant 1\}$.

In the extension of the corollary to Theorem 1 of [1], the replacement of $\mathbf{R}^{n}$ by $A$ causes some difficulty, and so full details of the proof are given. 
THEOREM 2. Under the hypotheses of Theorem 1 , there is a positive $t_{0}$ such that

$$
m\left(\left\{x: t^{\alpha} w(x, t)>1\right\}\right) \geqslant C_{n}\left(\mu(A) t^{\alpha+1}\right)^{n /(n+1)}
$$

whenever $0<t \leqslant t_{0}$.

Proof. Since $\mu$ is regular, we can find a compact subset of $K$ of $A$ such that $\mu(K) \geqslant \mu(A) / 2$, and an open superset $V$ of $K$ such that $\mu(V) \leqslant 2 \mu(A)$ (ignoring the trivial case where $\mu(A)=0$ ). Now let $\nu$ be a point mass at $x_{0} \in A$ with $\nu\left(\mathbf{R}^{n}\right)=2 \mu(A)$. Then, inserting extra subscripts to distinguish between the two measures, we have $\omega_{A, \nu}(r)=2 \mu(A)$ for all $r>0$, and hence $\omega_{K, \mu}(r) \leqslant \mu(V) \leqslant$ $2 \mu(A)=\omega_{A, \nu}(r)$ for all $r<r_{0}$, where $r_{0}$ is the distance between $K$ and $\mathbf{R}^{n} \backslash V$ in the $d$-metric. Next, $\delta=\delta_{\alpha, A, \nu}(t)$ satisfies

$$
\gamma_{n} t^{\alpha+1} \delta^{-n-1} 2 \mu(A)=1,
$$

so that

$$
\delta=\left(2 \gamma_{n} \mu(A) t^{\alpha+1}\right)^{1 /(n+1)} .
$$

Therefore $\delta \rightarrow 0$ as $t \rightarrow 0$, so that we can find $t_{1}>0$ such that $\delta<r_{0}$ whenever $0<t \leqslant t_{1}$. Hence there are values of $r$ less than $r_{0}$ such that

$$
\gamma_{n} t^{\alpha+1} r^{-n-1} \omega_{K, \mu}(r) \leqslant \gamma_{n} t^{\alpha+1} r^{-n-1} \omega_{A, \nu}(r) \leqslant 1 .
$$

It follows that, for $t \leqslant t_{1}$, we have

$$
\delta_{\alpha, K, \mu}(t)=\inf \left\{r<r_{0}: \gamma_{n} t^{\alpha+1} r^{-n-1} \omega_{K, \mu}(r) \leqslant 1\right\} \leqslant \delta_{\alpha, A, \nu}(t) .
$$

Theorem 1 now implies that, for all sufficiently small $t$,

$$
\begin{aligned}
m\left(\left\{x: t^{\alpha} w(x, t)>1\right\}\right) & \geqslant C_{n} \mu(K) t^{\alpha+1} / \delta_{\alpha, K, \mu}(t) \\
& \geqslant C_{n} \mu(A) t^{\alpha+1} / \delta_{\alpha, A, \nu}(t) \\
& =C_{n}\left(\mu(A) t^{\alpha+1}\right)^{n /(n+1)} .
\end{aligned}
$$

By considering the case where $\mu$ is a point mass at the origin, it is easy to verify, by direct calculation, that $t^{(\alpha+1) n /(n+1)}$ can be the exact rate of decrease of $m\left(\left\{x: t^{\alpha} w(x, t)>1\right\}\right)$ as $t \rightarrow 0$. The advantage of Theorem 2 over Theorem 1 is that it does not mention $\delta_{\alpha, A}$, which may be defined only implicitly.

One consequence of the use of an arbitrary Borel set $A$, rather than $\mathbf{R}^{n}$, in the above results, is the following condition for absolute continuity.

THEOREM 3. If $w$ is the Poisson integral of $\mu$, and

$$
\liminf _{t \rightarrow 0} t^{-n /(n+1)} m(\{x: w(x, t)>1\})=0,
$$

then $\mu$ is absolutely continuous with respect to $m$. 
Proof. By Theorem 2, if (1) holds (with $\alpha=0$ ) $\mu$-a.e. on a Borel set $A$, then

$$
\liminf _{t \rightarrow 0} t^{-n /(n+1)} m(\{x: w(x, t)>1\}) \geqslant C_{n} \mu(A)^{n /(n+1)} .
$$

Therefore our hypothesis implies that (1) can hold only on sets of $\mu$-measure zero.

By the Lebesgue Decomposition Theorem, we can write $\mu=\mu_{a}+\mu_{s}$, where $\mu_{a}$ and $\mu_{s}$ are non-negative measures, $\mu_{a}$ is absolutely continuous and $\mu_{s}$ is singular (with respect to $m$ ). By Lemma 6.1 of [4], we have

$$
\mu_{s}(\bar{Q}(x, r)) r^{-n} \rightarrow \infty \quad \text { as } r \rightarrow 0
$$

$\mu_{s}$-a.e. on $\mathbf{R}^{n}$. Therefore (1) holds (with $\alpha=0$ ) at every point of a set $E$ with $\mu_{s}\left(\mathbf{R}^{n} \backslash E\right)=0$. But, from above, $\mu(E)=0$, so that $\mu_{s}(E)=0$ and hence $\mu_{s}$ is null.

As an application of Theorem 3, we give a variant of the well-known result that, if $v$ is continuous on $\mathbf{R}^{n} \times\left[0, \infty\left[\right.\right.$, non-negative and harmonic on $\left.\mathbf{R}^{n} \times\right] 0, \infty[$, and $v(\cdot, 0)=0$, then $v(x, t) \equiv C t$ for some constant $C \geqslant 0$.

THEOREM 4. Let $v$ be non-negative and harmonic on $\left.\mathbf{R}^{n} \times\right] 0, \infty[$. If

$$
\liminf _{t \rightarrow 0} v(x, t)=0
$$

m-a.e. on $\mathbf{R}^{n}$, and

$$
\liminf _{t \rightarrow 0} t^{-n /(n+1)} m(\{x: v(x, t)>1\})=0,
$$

then there is a constant $C$ such that $v(x, t)=C t$ throughout $\left.\mathbf{R}^{n} \times\right] 0, \infty[$.

Proof. Since $v$ is non-negative and harmonic, we can write

$$
v(x, t)=C t+w(x, t)
$$

for all $\left.(x, t) \in \mathbf{R}^{n} \times\right] 0, \infty[$, where $C$ is a non-negative constant and $w$ is the Poisson integral of a non-negative measure $\mu$ (see [5]). Since $w \leqslant v$, we can apply Theorem 3 to $w$ and deduce that $\mu$ is absolutely continuous with respect to $m$. In view of the Fatou theorem [7, Theorem 1] and of Lebesgue's theorem on differentiation of measures, $v(x, 0+)$ exists $m$-a.e. and $d \mu(x)=v(x, 0+) d x$. It follows that $w=0$.

\section{The parabolic analogue of Theorem 1}

We now consider the case of a weakly coupled parabolic system of second-order, linear, partial differential equations:

$$
\frac{\partial u_{k}}{\partial t}=\sum_{i, j=1}^{n} a_{i j}^{k}(x, t) \frac{\partial^{2} u_{k}}{\partial x_{i} \partial x_{j}}+\sum_{i=1}^{n} b_{i}^{k}(x, t) \frac{\partial u_{k}}{\partial x_{i}}+\sum_{l=1}^{N} c_{l}^{k}(x, t) u_{l}
$$


for $k=1, \ldots, N$ and $(x, t) \in \mathbf{R}^{n} \times 10, T[$. We require the following properties of the solutions of (3). Conditions on the coefficients which guarantee these properties are given in [3] or [4], where further details and references can be found.

(i) There exists a fundamental solution (or matrix) $\left\{\Gamma_{i j}(x, t ; y, s)\right\}_{N \times N}$, defined and non-negative for all $(x, t),(y, s) \in \mathbf{R}^{n} \times[0, T]$ such that $t>s$, which satisfies

$$
\kappa(t-s)^{-n / 2} \exp \left(-\lambda\|x-y\|^{2} / 4(t-s)\right) \leqslant \Gamma_{i i}(x, t ; y, s)
$$

for $i=1, \ldots, N$, where $\kappa$ and $\lambda$ are positive constants. (See Section 4 of [4].)

(ii) A function $u=\left(u_{1}, \ldots, u_{N}\right)$ is a non-negative solution of (3) if and only if there are non-negative Borel measures $\mu_{1}, \ldots, \mu_{N}$ on $\mathbf{R}^{n}$ such that

$$
u_{i}(x, t)=\int_{\mathbf{R}^{n}} \sum_{j=1}^{N} \Gamma_{i j}(x, t ; y, 0) d \mu_{j}(y)
$$

for $i=1, \ldots, N$ and $\left.(x, t) \in \mathbf{R}^{n} \times\right] 0, T[$, where the integrals are finite. (See Theorem 1 of [3] and Theorem 5.1 of [4].) In the sequel, we shall always assume that our measures are such that the integrals in (4) are finite.

(iii) If $0 \leqslant \alpha<n$, (4) holds, and

$$
\mu_{i}(\bar{Q}(x, r)) r^{\alpha-n} \rightarrow \infty \quad \text { as } r \rightarrow 0
$$

for some $i$ and some $x \in \mathbf{R}^{n}$, then

$$
t^{\alpha / 2} u_{i}(x, t) \rightarrow \infty \quad \text { as } t \rightarrow 0 .
$$

(This follows easily from Corollary 6.1 of [4].)

We now develop analogues of Lemma 2 and Theorem 1. It is important for our applications in Section 5 that we keep track of the values of our various constants, and that these should be the best that our methods allow. We let $\chi$ denote an arbitrary, fixed number greater than 1 .

Lemma 4. Suppose that $d(x, z) \leqslant r$, that $0<t \leqslant \chi r^{2}$, and that (4) holds. Then

$$
u_{i}(x, t) \geqslant \pi_{n, \kappa} \exp \left(-\lambda n r^{2} / t\right) \mu_{i}(\bar{Q}(z, r)) r^{-n},
$$

where $\lambda$ and $\kappa$ are the same as in (i), and where $\pi_{n, \kappa}=\kappa \chi^{-n / 2}$.

Proof. If $d(x, z) \leqslant r$ and $y \in \bar{Q}(z, r)$, then

$$
\|x-y\| \leqslant n^{1 / 2} d(x, y) \leqslant 2 n^{1 / 2} r .
$$


It follows from (i) and (4) that, if $0<t \leqslant \chi r^{2}$, then

$$
\begin{aligned}
u_{i}(x, t) & \geqslant \int_{\mathbf{R}^{n}} \Gamma_{i i}(x, t ; y, 0) d \mu_{i}(y) \\
& \geqslant \kappa t^{-n / 2} \int_{\bar{Q}(z, r)} \exp \left(-\lambda\|x-y\|^{2} / 4 t\right) d \mu_{i}(y) \\
& \geqslant \kappa\left(\chi r^{2}\right)^{-n / 2} \int_{\bar{Q}(z, r)} \exp \left(-\lambda n r^{2} / t\right) d \mu_{i}(y) \\
& =\pi_{n, \kappa} \exp \left(-\lambda n r^{2} / t\right) \mu_{i}(\bar{Q}(z, r)) r^{-n} .
\end{aligned}
$$

For each integer $i(1 \leqslant i \leqslant N)$ and Borel set $A$, we denote the modulus of continuity of $\mu_{i}$ over $A$ by $\omega_{A, i}$.

Let $\pi_{n, \kappa}$ be the same as in (6). If $0 \leqslant \alpha<n$, and if $A$ is a Borel set, we put

$$
\rho_{A}(t)=\rho_{\alpha, A, i}(t)=\inf \left\{r: \pi_{n, \kappa} t^{\alpha / 2} \exp \left(-\lambda n r^{2} / t\right) \omega_{A, i}(r) r^{-n} \leqslant 1\right\}
$$

for all $t \in] 0, T[$.

THEOREM 5. Suppose that $0 \leqslant \alpha<n$, that $u_{i}$ is given by (4), and that (5) holds $\mu_{i}$-a.e. on a Borel set $A$ with $\mu_{i}(A)<\infty$. Then there exists $t_{0}>0$ such that

$$
m\left(\left\{x: t^{\alpha / 2} u_{i}(x, t)>1\right\}\right) \geqslant \theta_{n, \alpha} \mu_{i}(A) t^{\alpha / 2} \exp \left(-\lambda n \rho_{\alpha, A, i}(t)^{2} / t\right)
$$

whenever $0<t \leqslant t_{0}$, where $\theta_{n, \kappa}=(2 / 3)^{n} \chi^{-(n+2) / 2} \kappa$.

Proof. It follows from Lemma 1 , and the regularity of $\mu_{i}$, that there is a compact set $K$ such that $\mu_{i}(K) \geqslant \mu_{i}(A) / \chi$ and

$$
\mu_{i}(\bar{Q}(x, r)) r^{\alpha-n} \rightarrow \infty \quad \text { as } r \rightarrow 0
$$

uniformly for $x$ in $K$. Therefore we can find $t_{0}>0$ such that, whenever $0<r \leqslant t_{0}^{1 / 2}$, we have

$$
\pi_{n, \kappa} \mu_{l}(\bar{Q}(x, r)) r^{\alpha-n} \geqslant e^{\lambda n} \quad \text { for all } x \in K .
$$

Fix $\left.t \in] 0, t_{0}\right]$. For every $x \in K$, put

$$
\rho(x, t)=\rho_{\alpha, i}(x, t)=\inf \left\{r: \pi_{n, \kappa} t^{\alpha / 2} \exp \left(-\lambda n r^{2} / t\right) \mu_{i}(\bar{Q}(x, r)) r^{-n} \leqslant 1\right\} .
$$

Then

$$
\rho(x, t) \leqslant \rho_{A}(t)
$$

for all $x \in K$. Furthermore, given $x$, there is a sequence $\left\{r_{k}\right\}$ which decreases to $\rho(x, t)$ and which is such that

$$
\pi_{n, \kappa} t^{\alpha / 2} \exp \left(-\lambda n r_{k}^{2} / t\right) \mu_{i}\left(\bar{Q}\left(x, r_{k}\right)\right) r_{k}^{-n} \leqslant 1
$$

for all $k$. Making $k \rightarrow \infty$, we deduce that

$$
\pi_{n, k} \alpha^{\alpha / 2} \exp \left(-\lambda n \rho(x, t)^{2} / t\right) \mu_{i}(\bar{Q}(x, \rho(x, t))) \rho(x, t)^{-n} \leqslant 1 \text {. }
$$


Next, if $0<r<t^{1 / 2}$, then it follows from (7) that

$$
\begin{aligned}
\pi_{n, \kappa} t^{\alpha / 2} \exp \left(-\lambda n r^{2} / t\right) \mu_{i}(\bar{Q}(x, r)) r^{-n} & \geqslant t^{\alpha / 2} \exp \left(-\lambda n r^{2} / t\right) r^{-\alpha} e^{\lambda n} \\
& >1 .
\end{aligned}
$$

Therefore, for all $x \in K$, we have

$$
t^{1 / 2} \leqslant \rho(x, t) .
$$

Since $K$ is compact, and since the family

$$
\{Q(x, \rho(x, t) / 3): x \in K\}
$$

of open cubes covers $K$, we can select a finite subfamily $\mathscr{F}$ which also covers $K$. By Lemma 3, $\mathscr{F}$ has a subfamily

$$
\left\{Q\left(x_{l}, \rho\left(x_{l}, t\right) / 3\right): l=1, \ldots, q\right\}
$$

of disjoint cubes such that

$$
K \subseteq \bigcup_{l=1}^{q} Q\left(x_{l}, \rho\left(x_{l}, t\right)\right)
$$

Therefore

$$
\mu_{i}(A) \leqslant \chi \mu_{i}(K) \leqslant \chi \sum_{t=1}^{q} \mu_{i}\left(Q\left(x_{l}, \rho\left(x_{i}, t\right)\right)\right) .
$$

It now follows from (9) and (8) that

$$
\begin{aligned}
\pi_{n, \kappa} t^{\alpha / 2} \mu_{i}(A) & \leqslant \chi \sum_{l=1}^{q} \exp \left(\lambda n \rho\left(x_{l}, t\right)^{2} / t\right) \rho\left(x_{l}, t\right)^{n} \\
& \leqslant \chi \exp \left(\lambda n \rho_{A}(t)^{2} / t\right) \sum_{l=1}^{q} \rho\left(x_{l}, t\right)^{n} .
\end{aligned}
$$

Therefore, because the cubes in the family (11) are disjoint, we obtain

$$
\begin{aligned}
(2 / 3)^{n} & \chi^{-1} \pi_{n, \kappa} t^{\alpha / 2} \mu_{i}(A) \exp \left(-\lambda n \rho_{A}(t)^{2} / t\right) \\
& \leqslant \sum_{l=1}^{q}\left(2 \rho\left(x_{l}, t\right) / 3\right)^{n} \\
& =\sum_{l=1}^{q} m\left(Q\left(x_{l}, \rho\left(x_{l}, t\right) / 3\right)\right) \\
& \leqslant m\left(\bigcup_{l=1}^{q} Q\left(x_{l}, \rho\left(x_{l}, t\right)\right)\right) .
\end{aligned}
$$

If $y$ belongs to the set whose measure is the last term in (12), then $y \in Q(z, \rho(z, t))$ for some $z \in K$. Since $d(y, z)<\rho(z, t)$, and since $(t / \chi)^{1 / 2}<\rho(z, t)$ by (10), we can choose $r$ such that $t<\chi r^{2}$ and $d(y, z)<r<\rho(z, t)$. Therefore, by Lemma 4 ,

$$
t^{\alpha / 2} u_{i}(y, t) \geqslant \pi_{n, \kappa^{\alpha}} \alpha^{\alpha / 2} \exp \left(-\lambda n r^{2} / t\right) \mu_{i}(\bar{Q}(z, r)) r^{-n}>1
$$


since $r<\rho(z, t)$. Therefore (12) implies that

$$
\theta_{n, \kappa} t^{\alpha / 2} \mu_{i}(A) \exp \left(-\lambda n \rho_{A}(t)^{2} / t\right) \leqslant m\left(\left\{y: t^{\alpha / 2} u_{i}(y, t)>1\right\}\right),
$$

as required.

\section{The parabolic version of Theorem 2}

Theorem 5 is analogous to Theorem 1, and a comparison of the two yields no real surprises. The situation is different, however, for Theorem 2 and its analogue, Theorem 6 below. In Theorem 2, the parameter $\alpha$ appears as a power of $t$, while $\mu(A)$ appears as a multiplicative factor; in Theorem $6, \alpha$ appears only as part of a multiplicative constant, and $\mu_{i}(A)$ is completely absent. We must therefore assume that $\mu_{i}(A)>0$.

THEOREM 6. Suppose that $0 \leqslant \alpha<n$, that $u_{i}$ is given by (4), and that (5) holds $\mu_{i}$-a.e. on a Borel set $A$ with $0<\mu_{i}(A)<\infty$. Then we can find $t_{0}>0$ such that

$$
m\left(\left\{x: t^{\alpha / 2} u_{i}(x, t)>1\right\}\right) \geqslant \Delta_{n, \lambda}(n-\alpha)^{n / 2}|t \log t|^{n / 2}
$$

whenever $0<t \leqslant t_{0}$, where $\Delta_{n, \lambda}=2^{n / 2} 3^{-n} \chi^{-n-3}(\lambda n)^{-n / 2}$.

Proof. Let $\nu$ be a point mass at $x_{0} \in A$ with $\nu(A)=\chi \mu_{i}(A)$, so that $\omega_{A, \nu}(r)=\chi \mu_{i}(A)$ for all $r>0$. The method for proving Theorem 6 is basically similar to that for Theorem 2 , and so we want to find $\rho=\rho_{\alpha, A, \nu}(t)$ which satisfies

$$
\chi \mu_{i}(A) \pi_{n, \kappa} t^{\alpha / 2} \exp \left(-\lambda n \rho^{2} / t\right) \rho^{-n}=1 \text {. }
$$

Since $\rho$ is defined only implicitly, we shall have to manage with an approximation to $\rho$ for small $t$.

Consider the function $\sigma$ defined by

$$
\sigma(\tau)=\left\{-(\tau / 2) \log \left(-a \tau^{(n-\alpha) / n} \log \tau\right)\right\}^{1 / 2}
$$

for all small positive $\tau$, where $\tau=t / \lambda$, and where

$$
a=(n-\alpha)\left\{2 n\left(\chi \mu_{i}(A) \pi_{n, \kappa} \lambda^{\alpha / 2}\right)^{2 / n}\right\}^{-1} \text {. }
$$

As $t \rightarrow 0$, we have

$$
\begin{aligned}
t^{\alpha / 2} \exp \left(-\lambda n \sigma^{2} / t\right) \sigma^{-n} \\
=(\lambda \tau)^{\alpha / 2} \exp \left(-n \sigma^{2} / \tau\right) \sigma^{-n} \\
=(\lambda \tau)^{\alpha / 2}\left(-a \tau^{(n-\alpha) / n} \log \tau\right)^{n / 2}\left\{-(\tau / 2) \log \left(-a \tau^{(n-\alpha) / n} \log \tau\right)\right\}^{-n / 2} \\
=\lambda^{\alpha / 2}\left\{2 a \log \tau / \log \left(-a \tau^{(n-\alpha) / n} \log \tau\right)\right\}^{n / 2} \\
\rightarrow \lambda^{\alpha / 2}\{2 a n /(n-\alpha)\}^{n / 2} \\
=\left(\chi \mu_{i}(A) \pi_{n, \kappa}\right)^{-1}
\end{aligned}
$$


thus

$$
\chi \mu_{i}(A) \pi_{n, \kappa} t^{\alpha / 2} \exp \left(-\lambda n \sigma^{2} / t\right) \sigma^{-n} \rightarrow 1
$$

as $t \rightarrow 0$. Put

$$
f(r, t)=\chi \mu_{i}(A) \pi_{n, \kappa} t^{\alpha / 2} \exp \left(-\lambda n r^{2} / t\right) r^{-n}
$$

for all $r>0$ and $t>0$, and put $\zeta(t)=\sigma(t / \lambda)$. Then

$$
f(\zeta(t), t) \rightarrow 1 \text { as } t \rightarrow 0,
$$

and

$$
f(\rho(t), t)=1 \text { for all sufficiently small } t,
$$

so that

$$
f(\zeta(t), t) \sim f(\rho(t), t) \text { as } t \rightarrow 0 .
$$

We assert that

$$
\zeta(t) \sim \rho(t) \text { as } t \rightarrow 0 .
$$

If (14) is false, then there is $\eta>0$ and a null sequence $\left\{t_{k}\right\}$ such that either

$$
\zeta\left(t_{k}\right)>(1+\eta) \rho\left(t_{k}\right) \text { or } \rho\left(t_{k}\right)>(1+\eta) \zeta\left(t_{k}\right)
$$

for all $k$. We suppose that the former is the case; the proof for the latter is similar. For each fixed $t$, the function $f(\cdot, t)$ is strictly decreasing, so that

$$
f\left(\zeta\left(t_{k}\right), t_{k}\right)<f\left((1+\eta) \rho\left(t_{k}\right), t_{k}\right)
$$

for all $k$. Therefore

$$
\begin{aligned}
\frac{f\left(\zeta\left(t_{k}\right), t_{k}\right)}{f\left(\rho\left(t_{k}\right), t_{k}\right)} & <(1+\eta)^{-n} \exp \left\{-\lambda n \rho\left(t_{k}\right)^{2}\left(\eta^{2}+2 \eta\right) / t_{k}\right\} \\
& <(1+\eta)^{-n}
\end{aligned}
$$

for all $k$, contrary to (13). Thus (14) is proved, so that

$$
\rho(t) \sim\left\{-(t / 2 \lambda) \log \left(-a(t / \lambda)^{(n-\alpha) / n} \log (t / \lambda)\right)\right\}^{1 / 2}
$$

as $t \rightarrow 0$.

We can now complete the proof. Since $\mu_{i}$ is regular, we can find a compact subset $K$ of $A$ such that $\mu_{i}(K) \geqslant \mu_{i}(A) / \chi$, and an open superset $V$ of $K$ such that $\mu_{i}(V) \leqslant \chi \mu_{i}(A)$. With $\nu$ as above, we have

$$
\omega_{K, i}(r) \leqslant \mu_{i}(V) \leqslant \chi \mu_{i}(A)=\omega_{A, \nu}(r)
$$

for all $r<r_{0}$, where $r_{0}$ is the distance between $K$ and $\mathbf{R}^{n} \backslash V$ in the $d$-metric. In view of (15), $\rho \rightarrow 0$ as $t \rightarrow 0$, so that we can find $t_{1}>0$ such that $\rho<r_{0}$ whenever $0<t \leqslant t_{1}$. Hence there are values of $r$ less than $r_{0}$ such that

$$
\begin{aligned}
\pi_{n, \kappa} t^{\alpha / 2} \exp \left(-\lambda n r^{2} / t\right) \omega_{K, i}(r) r^{-n} & \leqslant \pi_{n, \kappa} t^{\alpha / 2} \exp \left(-\lambda n r^{2} / t\right) \omega_{A, \nu}(r) r^{-n} \\
& \leqslant 1 .
\end{aligned}
$$


It now follows that

$$
\begin{aligned}
\rho_{\alpha, K, i}(t) & =\inf \left\{r<r_{0}: \pi_{n, k} t^{\alpha / 2} \exp \left(-\lambda n r^{2} / t\right) \omega_{K, i}(r) r^{-n} \leqslant 1\right\} \\
& \leqslant \rho_{\alpha, A, \nu}(t)=\rho(t) .
\end{aligned}
$$

Therefore, by Theorem 5 ,

$$
\begin{aligned}
m\left(\left\{x: t^{\alpha / 2} u_{i}(x, t)>1\right\}\right) & \geqslant \theta_{n, \kappa} \mu_{i}(K) t^{\alpha / 2} \exp \left(-\lambda n \rho_{\alpha, K, i}(t)^{2} / t\right) \\
& \geqslant \theta_{n, \kappa} x^{-1} \mu_{i}(A) t^{\alpha / 2} \exp \left(-\lambda n \rho(t)^{2} / t\right) .
\end{aligned}
$$

Since $f(\rho(t), t)=1$ for all sufficiently small $t$, it follows that

$$
m\left(\left\{x: t^{\alpha / 2} u_{i}(x, t)>1\right\}\right) \geqslant \epsilon_{n, \kappa} \chi^{-2} \pi_{n, \kappa}^{-1} \rho(t)^{n}=(2 / 3)^{n} \chi^{-3} \rho(t)^{n} .
$$

In view of (15) we have, as $t \rightarrow 0$,

$$
\begin{aligned}
\frac{\rho(t)^{2}}{|t \log t|} & \geqslant \frac{-(t / 2 \lambda) \log \left(-a(t / \lambda)^{(n-\alpha) / n} \log (t / \lambda)\right)}{-\chi t \log t} \\
& \rightarrow(n-\alpha) / 2 \chi \lambda n .
\end{aligned}
$$

Therefore, for all sufficiently small $t$, we have

$$
\rho(t)^{2} \geqslant(n-\alpha)|t \log t| / 2 \chi^{2} \lambda n,
$$

and hence

$$
m\left(\left\{x: t^{\alpha / 2} u_{i}(x, t)>1\right\}\right) \geqslant \Delta_{n, \lambda}(n-\alpha)^{n / 2}|t \log t|^{n / 2},
$$

as required.

The result of Theorem 6 is sharp for every value of $\alpha$, in the sense that $|t \log t|^{n / 2}$ can be the exact rate of decrease of $m\left(\left\{x: t^{\alpha / 2} u_{i}(x, t)>1\right\}\right)$ as $t \rightarrow 0$, at least for the case of the heat equation $(N=1)$. This can be verified by direct calculation by taking $\mu_{1}$ to be a point mass at the origin (since the lower estimate for $\Gamma_{i i}$ in (i) is then an identity). However, it is extremely unlikely that the constant $\Delta_{n, \lambda}$ is the best possible, and this is slightly unfortunate when we come to consider applications in the next section.

\section{Applications in the parabolic case}

The theorems of this section are parabolic analogues of Theorems 3 and 4 . However, the precise forms of the parabolic results are significantly different to those of their harmonic counterparts, due to the absence of $\mu_{i}(A)$ from the conclusion of Theorem 6 . 
THEOREM 7. If $u_{i}$ is given by (4), and if

$$
\liminf _{t \rightarrow 0}|t \log t|^{-n / 2} m\left(\left\{x: u_{i}(x, t)>1\right\}\right)<(2 / 9 \lambda)^{n / 2},
$$

then $\mu_{i}$ is absolutely continuous with respect to $m$.

Proof. Condition (16) implies that there is a null sequence $\left\{t_{k}\right\}$ such that the sequence $\left\{\left|t_{k} \log t_{k}\right|^{-n / 2} m\left(\left\{x: u_{i}\left(x, t_{k}\right)>1\right\}\right)\right\}$ converges to a limit $\phi<$ $(2 / 9 \lambda)^{n / 2}$. Choose $\chi_{0}>1$ such that $\phi<\chi_{0}^{-n-3}(2 / 9 \lambda)^{n / 2}$. Then, for any $t_{0}>0$, we can find $k$ such that $t_{k}<t_{0}$, and

$$
m\left(\left\{x: u_{i}\left(x, t_{k}\right)>1\right\}\right)<\chi_{0}^{-n-3}(2 / 9 \lambda)^{n / 2}\left|t_{k} \log t_{k}\right|^{n / 2} .
$$

Therefore Theorem 6 (with $\alpha=0$ and $\chi=\chi_{0}$ ) implies that (5) does not hold on any Borel set $A$ with $0<\mu_{i}(A)<\infty$. Hence (5) can hold (with $\alpha=0$ ) only on sets of $\mu_{i}$-measure zero, and the proof can be completed by the argument in the last paragraph of the proof of Theorem 3.

The principal difference between Theorem 7 and its harmonic counterpart is that the lower limit in (16) can be positive. The constawnt $(2 / 9 \lambda)^{n / 2}$ is derived from the constants in the earlier theorems, and this is why it was important to keep them as large as possible; but it is most unlikely to be the best possible. If we reconsider the case of the heat equation, with $\mu_{1}$ a point mass, which showed that the rate of decrease $|t \log t|^{n / 2}$ is exact, we get

$$
\lim _{t \rightarrow 0}|t \log t|^{-n / 2} m\left(\left\{x: u_{1}(x, t)>1\right\}\right)=v_{n}(2 n / \lambda)^{n / 2},
$$

where $v_{n}=\pi^{n / 2} / \Gamma((n+2) / 2)$ is the volume of the unit ball in $\mathbf{R}^{n}$. This gives an upper bound for the best constant.

THEOREM 8. Let $u=\left(u_{1}, \ldots, u_{N}\right)$ be a non-negative solution of (3) on $\mathbf{R}^{n} \times$ $10, T[$. If, for every $i$, we have

$$
\liminf _{t \rightarrow 0} u_{i}(x, t)=0
$$

m-a.e. on $\mathbf{R}^{n}$, and (16) holds, then $u=0$ on $\mathbf{R}^{n} \times 10, T[$.

Proof. By (ii), each $u_{i}$ is given by (4). Since (16) holds for all $i$, Theorem 7 implies that each $\mu_{i}$ is absolutely continuous with respect to $m$. Since (17) holds for all $i$, the Fatou theorem [4, Theorem 3.1] implies that each $\mu_{i}$ is null. 


\section{Added in proof}

The constants in the parabolic case can be improved by using balls instead of cubes throughout. Hence, in particular, Theorem 7 holds with $(2 / 9 \lambda)^{n / 2}$ replaced by $v_{n}(n / 18 \lambda)^{n / 2}$.

\section{References}

[1] P. Ahern, 'The Poisson integral of a singular measure', Canad. J. Math. 35 (1983), 735-749.

[2] D. H. Armitage, 'Normal limits, half-spherical means and boundary measures of half-space Poisson integrals', Hiroshima Math. J. 11 (1981), 235-246.

[3] J. Chabrowski and N. A. Watson, 'Properties of solutions of weakly coupled parabolic systems', J. London Math. Soc. 23 (1981), 475-495.

[4] J. Chabrowski, 'Representation theorems for parabolic systems', J. Austral. Math. Soc. Ser. A 32 (1982), 246-288.

[5] A. Dinghas, 'Über positive harmonische Funktionen in einem Halbraum', Math. Z. 46 (1940), 559-570.

[6] T. M. Flett, 'On the rate of growth of mean values of holomorphic and harmonic functions', Proc. London Math. Soc. 20 (1970), 749-768.

[7] F. W. Gehring, 'The Fatou theorem for functions harmonic in a half-space', Proc. London Math. Soc. 8 (1958), 149-160.

[8] C. A. Rogers and S. J. Taylor, 'Functions continuous and singular with respect to a Hausdorff measure', Mathematika 8 (1961), 1-31.

\section{Department of Mathematics}

University of Canterbury

Christchurch

New Zealand 